American Journal of Applied Sciences 4 (10): 736-740, 2007

ISSN 1546-9239

(C) 2007 Science Publications

\title{
MAI Cancellation in DS/CDMA using a new Approach on WDS
}

\author{
${ }^{1}$ Y. Jabrane, ${ }^{1}$ R.Iqdour, ${ }^{1}$ B. Ait Es said and ${ }^{2}$ N.Naja \\ ${ }^{1}$ Department of physics, Cadi Ayyad University, Faculty of Sciences Semlalia, Avenue Prince \\ My Abdellah, P.O. Box 2390, 40001, Marrakesh, Morocco \\ ${ }^{2}$ National Institute of Poste and Telecommunications, Av Allal Al Fassi- Madinat Al Irfane \\ Rabat-Instituts, Morocco
}

\begin{abstract}
The steeping chip weighting waveforms are used in Multiple Access Interference cancellation by emphasizing the received spreading signal, therefore, that allows to solve the problem of orthogonality for the chip waveforms. The goal of this study was to elaborate a useful method based on fuzzy systems to determine the despreading sequences weighted by the steeping chip weighting waveforms for Direct Sequence Code Division Multiple Access (DS/CDMA). The validity of the proposed method has been tested by numerical examples for an Additive White Gaussian Noise channels and shows that the parameter values of the chip weighting waveforms are good and the Bit Error Rate (BER) performance of the system does not undergone any degradation.
\end{abstract}

Key words: Direct sequence- code division multiple access- weighted despreading sequences- fuzzy systems- multiple access interference

\section{INTRODUCTION}

In a DS/CDMA system, the biggest problem limiting its performances and capacity is due to interference produced by multiple access of several users in the channel ${ }^{[1-2]}$ (Multiple Access Interference MAI). Several studies have been made in the goal to reject MAI, but are disadvantaged by their computational complexity in the number of users and their requiring knowledge of delays, amplitudes and modulation waveforms of the desired user and the interfering users.

In a previous work $^{[3-4]}$, a method has been proposed to Weight Despreading Sequences (WDS) by stepping chip weighting waveforms, with the purpose to the MAI cancellation. The despreading sequences were expressed according to one parameter. This parameter has been adjusted in order to maximize the signal to interference plus noise ratio (SINR), nevertheless, for each spreading code the calculation of optimal values of this parameter which maximize SINR while varying the signal to noise ratio (SNR) is not so easy.

In this study, we propose a new method based on fuzzy systems to determine the WDS for a DS/CDMA system. Our goal is to reduce the complexity calculation of the optimal values of the parameter for each SNR by using the learning ability and the high-speed computational capacity features of fuzzy systems.
Model statement: We consider the system described by Huang and Tung-Sang ${ }^{[3]}$, the transmitted signal relative to the $k$ th user, given by:

$S_{k}(t)=\sqrt{2 P} b_{k}(t) a_{k}(t) \cos \left(\omega_{c} t+\Theta_{k}\right)$

Where $\mathrm{P}$ and $\omega_{c}$, common to all users, are the transmitted power and the carrier frequency, respectively, $\Theta_{k}$ is a random phase. $b_{k}(t)$ is a binary data signal and $a_{k}(t)$ is the spreading code, which have respectively $T_{b}$ and $T_{c}$ as durations where $T_{b}=N T_{c}$ and $N$ is the period of the spreading sequence, $a_{k}(t)$ and $b_{k}(t)$ are given by:

$a_{k}(t)=\sum_{-\infty}^{\infty} a_{j}^{(k)} P_{T_{c}}\left(t-j T_{c}\right)$

$b_{k}(t)=\sum_{-\infty}^{\infty} b_{j}^{(k)} P_{T_{c}}\left(t-j T_{b}\right)$

Thus, the received signal $r(t)$ at the base station is given by:

$$
\begin{aligned}
& r(t)=\sum_{k=1}^{K} S_{k}\left(t-\tau_{k}\right)+n(t) \\
& =\sqrt{2 P} \sum_{k=1}^{K} a_{k}\left(t-\tau_{k}\right) b_{k}\left(t-\tau_{k}\right) \cos \left(\omega_{c} t+\Phi_{k}\right)+n(t)
\end{aligned}
$$

$\mathrm{K}$ is the total number of active users, $\tau_{k}$ and $\Phi_{k}$ are random time delays and phases, respectively, which are related by: $\Phi_{k}=\Theta_{k}-\omega_{c} \tau_{k}$, for $1 \leq k \leq K . n(t)$ is an Additive White Gaussian

Corresponding Author: Y. Jabrane, Department of Physics, Cadi Ayyad University, Faculty of Sciences Semlalia Avenue Prince My Abdellah, P.O. Box 2390, 40001, Marrakesh, Morocco 
Noise (AWGN) with tow-sided power spectral density $\underline{N_{0}}$. The weighted despreading sequence for the $k$ th reeeiver is given by ${ }^{[3]}$ :

$\hat{a}_{k}(t)=\sum_{j=-\infty}^{\infty} \begin{array}{rrr}a & (k) & j \\ a & j\end{array}\left(t-j T_{c} /\left\{\begin{array}{ll}(k) & (k) \\ c & c \\ j & j+1\end{array}\right\}\right)$

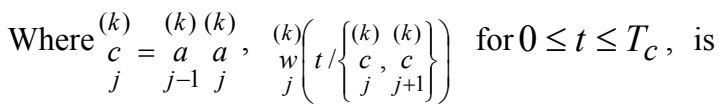
the $j$ th chip weighting waveforms for the $k$ th receiver conditioned on the status of three consecutive

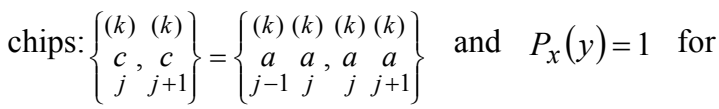
$0 \prec y \prec x$ and 0 otherwise. The $j$ th chip conditional weighting waveforms for the $k$ th receiver is defined by $^{[3]}$ :

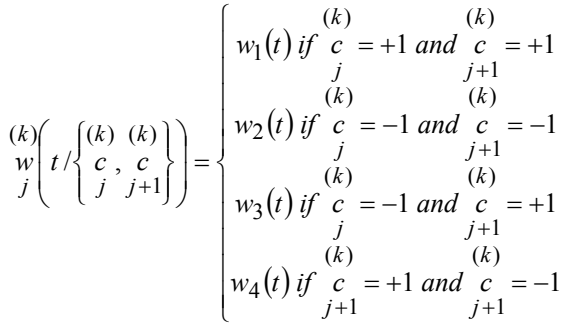

The elements of the chip weighting waveform vector $\left\{w_{1}(t), w_{2}(t), w_{3}(t), w_{4}(t)\right\}$ are given by:

$w_{1}(t)=L(\varepsilon) P_{T c}(t)$

$w_{2}(t)=P_{T c}(t)-[1-L(\varepsilon)] P_{T c-2 T \Delta}\left(t-T_{\Delta}\right)$

$w_{3}(t)=P_{T \Delta}(t)+L(\varepsilon) P_{T c-T \Delta}\left(t-T_{\Delta}\right)$

$w_{4}(t)=L(\varepsilon) P_{T c-T \Delta}(t)+P_{T c}(t)-P_{T c-T \Delta}(t)$

Where $T_{\Delta} \in\left(0, T_{c} / 2\right], \varepsilon=T_{c} / T_{\Delta} \in[2, \infty)$ is the parameter of the stepping chip weighting waveforms and $L(\varepsilon) \in[0,1]$ is a monotonically decreasing function with $\varepsilon:{ }_{L}(\varepsilon)=\left[C\left(\frac{\varepsilon}{2}-1\right)+1\right]^{-1}$, where the constant $\mathrm{C}$ is chosen equal to 10 . We assume that $\left(\tau_{i}=0\right.$ and $\left.\Phi_{i}=0\right)$ for the $i$ th user, the $\operatorname{SINR}_{i}$ Conditioned on $\left\{\begin{array}{l}(i) \\ c \\ j\end{array}\right\}$ is given by ${ }^{[3]}$ :

$\operatorname{SINR}_{i}=\left\{\frac{\varepsilon\left[2 \chi+(\varepsilon-2 \chi) L(\varepsilon)^{2}\right]}{2 k_{b}[2 \chi+(\varepsilon-2 \chi) L(\varepsilon)]^{2}}+\frac{(K-1) \mathrm{E}\left(\Gamma\left\{\begin{array}{c}i \\ c \\ j\end{array}\right\}, \varepsilon\right)}{2 \varepsilon N[2 \chi+(\varepsilon-2 \chi) L(\varepsilon)]^{2}}\right\}^{-1}$

Where: $k_{b}=E_{b} / N_{0}, E_{b}=P T_{b}, \chi=\hat{N}_{i} / N, \hat{N}_{i}$ is a random variable which represents the number of occurrences of $\underset{\substack{i \\ j}=-1}{i}$ for all $j \in[0, N-1]$ and the term $\mathrm{E}\left(\Gamma\left\{\begin{array}{l}i \\ c, \varepsilon \\ j\end{array}\right\}\right)$ is given by:

$$
\begin{aligned}
& \stackrel{(i)}{\Gamma}[-1,-1,-1\}\left[\frac{8}{3}+4(\varepsilon-2) L(\varepsilon)+\frac{(\varepsilon-2)^{2}(4+\varepsilon) L^{2}(\varepsilon)}{3}\right]+
\end{aligned}
$$

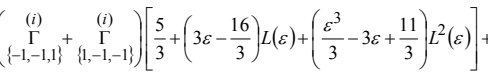

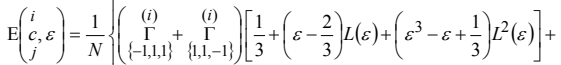

$$
\begin{aligned}
& \underset{\{-1,1,-1\}}{\stackrel{(i)}{\Gamma}}\left[\frac{2}{3}+2\left(\varepsilon-\frac{2}{3}\right) L(\varepsilon)+\left(\varepsilon^{3}-2 \varepsilon+\frac{2}{3}\right) L^{2}(\varepsilon)\right] \\
& \left.\underset{\{1,-1,1\}}{\stackrel{(i)}{\Gamma}}\left[\frac{2}{3}+\left(2 \varepsilon-\frac{8}{3}\right) L(\varepsilon)+\left(\frac{\varepsilon^{3}}{3}-2 \varepsilon+2\right) L^{2}(\varepsilon)\right]+\varepsilon^{3} L^{2}(\varepsilon) \underset{\{1,1,1\}}{\stackrel{(i)}{\Gamma}}\right\}
\end{aligned}
$$

Where $\stackrel{(i)}{\Gamma}$ is the number of occurrences of $\left\{\begin{array}{ccc}(i) & (i) & (i) \\ c & c & c \\ j-1 & j & j+1\end{array}\right\}=\left\{v_{1}, v_{2}, v_{3}\right\}$ for all $j \in[0, N-1]$ in the $i$ th WDS. Each element of $\left\{v_{1}, v_{2}, v_{3}\right\}$ takes a value +1 or -1 with equal probability. It is obvious that $\sum\left\{v_{1}, v_{2}, v_{3}\right\}_{\left\{v_{1}, v_{2}, v_{3}\right\}}^{\stackrel{(i)}{\Gamma}}=N \cdot$

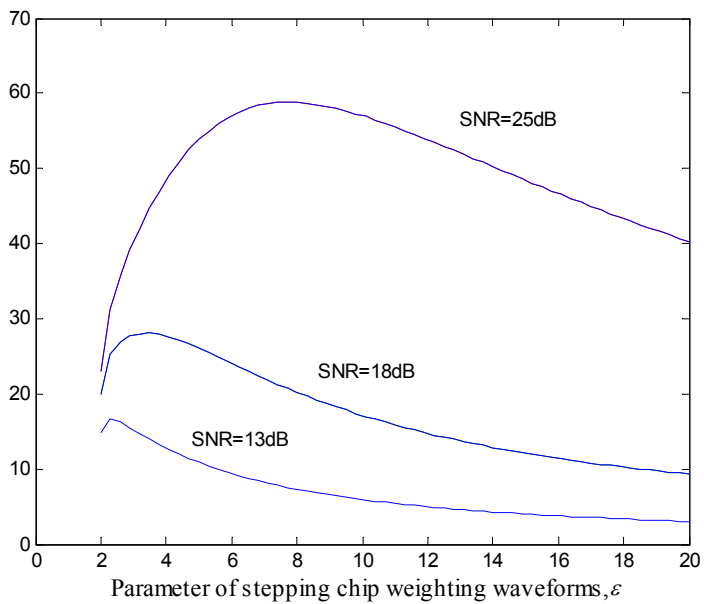

Fig. 1: SINR versus the parameter $\varepsilon$ for various values SNR when $\mathrm{K}=9$

In Fig. 1, SINR is plotted versus $\varepsilon$ for different values of $k_{b}=S N R$ when $\mathrm{K}=9$. In the simulation, we take the following values:

$$
\begin{aligned}
& N=63, \hat{N}_{i}=34, \underset{\{-1,-1,-1\}}{\stackrel{(i)}{\Gamma}}=6, \stackrel{(i)}{\Gamma}+\stackrel{(i)}{\Gamma} \\
& =20, \stackrel{(i)}{\Gamma}+\underset{\{-1,1,1\}}{\stackrel{(i)}{\Gamma}}=6, \stackrel{(i)}{\Gamma}=20, \\
& \stackrel{(i)}{\Gamma}=6 \text { and } \stackrel{(i)}{\Gamma}=5 \text {. }
\end{aligned}
$$

As can be seen from the Fig. 1, the values of the parameter $\varepsilon$ should be tuned to its optimal for different values of $k_{b}=S N R$, so the corresponding optimal values of $\varepsilon$ to $\mathrm{SNR}=13 \mathrm{~dB}, \mathrm{SNR}=18 \mathrm{~dB}$, $\mathrm{SNR}=25 \mathrm{~dB}$ are respectively nearly equal to $2.25,3.2$ 
and 8 . That allows us to reduce the bit error rate in detection $^{[3]}$ given by:

$$
B E R=\operatorname{erfc}(\sqrt{\max (\operatorname{SINR})})
$$

It is remarkable from eq. (8) and eq. (9) that it is not easy to calculate the optimal values of $\varepsilon$ for each code in a given code set.

Fuzzy systems based determination: TakagiSugeno fuzzy systems ${ }^{[5]}$ form a very special class of fuzzy systems because the conclusion of each rule is crisp (not a fuzzy set). A typical single antecedent fuzzy rule in a Takagi-Sugeno model of order $\mathrm{d}$ has the form: $R_{k}$ if $x_{t}$ is $A_{k}$ then:

$\hat{y}_{t, k}=P_{k}^{(d)}\left(x_{t}\right)$

for $\mathrm{k}=1,2, \ldots$, c. Where $x_{t}$ is the input variable $\left(x_{t} \in \mathfrak{R}^{n}\right), A_{k}$ is a fuzzy set of $R_{k}$ and $P_{k}^{(d)}\left(x_{t}\right)$ is a polynomial of order $\mathrm{d}$ in the components $x_{t, i}$ of $x_{t}$. In the sequel, we will suppose $d=1$. For convenience, we will write the conclusion of rule $R_{k}$ relatively to input $x_{t}$ as:

$\hat{y}_{t, k}=x_{t}{ }^{\prime} \beta_{k}$

Where,

$\beta_{k}=\left(\beta_{1}, \ldots, \beta_{n}\right)^{\prime}$

An intercept is allowed in the conclusion $\hat{y}_{t, k}$ if we suppose $x_{t, 1}=1$ (bias term). Output $\hat{y}_{t}$ relative to input $x_{t}$ obtained after aggregating a set of c TS rules can be written as a weighted sum of the individual conclusions:

$$
\begin{gathered}
\hat{y}_{t}=\sum_{k=1}^{c} \prod_{k}\left(x_{t}\right) \hat{y}_{t, k} \\
\prod_{k}\left(x_{t}\right)=\frac{\mu_{A_{k}}\left(x_{t}\right)}{\sum_{j=1}^{c} \mu_{A_{j}}\left(x_{t}\right)}
\end{gathered}
$$

Where $\mu_{A_{k}}$ is the membership function related to the fuzzy set $A_{k}$. The setting up of a fuzzy system requires two types of tuning ${ }^{[6]}$ :

Structural tuning: The number of the fuzzy rules c and the antecedent fuzzy sets $\left(A_{k}, \mathrm{k}=1, \ldots, \mathrm{c}\right)$ are identified. Many techniques are available in the literature. In this study we used an exhaustive method, based on the use of the Gustafson-Kessel (GK) fuzzy clustering algorithm, which consists to initialise and to adjust the parameters for each selected structure, while starting with a system with two rules $(c=2)$. The optimal number of the clusters $\mathrm{c}$ is that which gives a minimal value of the Root Mean Squares Error (RMSE) validity criterion.
Parametric tuning: The model parameters (linear and non-linear) are estimated. The goal of the parameters optimisation is to find the "best»" approximation $\hat{y}_{t}$ to the measured output $y_{t}$. The linear parameters $\beta_{k}$ are identified using the Weighted Least Square (WLS) algorithm, while the Levenberg-Marquardt (LM) algorithm is using to estimate the non linear parameters $\left(S_{k}\right.$ and $\left.m_{k}\right)$. The TS Fuzzy model employed has eight inputs and one output: Seven of the inputs are bound directly to the used code ${ }^{[7-8]}$ :

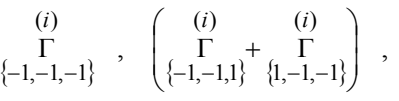

$$
\begin{aligned}
& \left(\stackrel{(i)}{\Gamma}+\stackrel{(i)}{\Gamma}+\stackrel{(i)}{\Gamma} \underset{\{-1,1,1\}}{\Gamma} \underset{\{1,1,-1\}}{\stackrel{(i)}{\Gamma}}, \underset{\{-1,1,-1\}}{\stackrel{(i)}{\Gamma}} \text { and } \hat{N}_{i} .\right.
\end{aligned}
$$

The last input is $k_{b}$ and the output of the TS fuzzy model is $\varepsilon_{f s}$. The $k_{b}$ values of training data have been taken from the range of $[0,25] d B$.
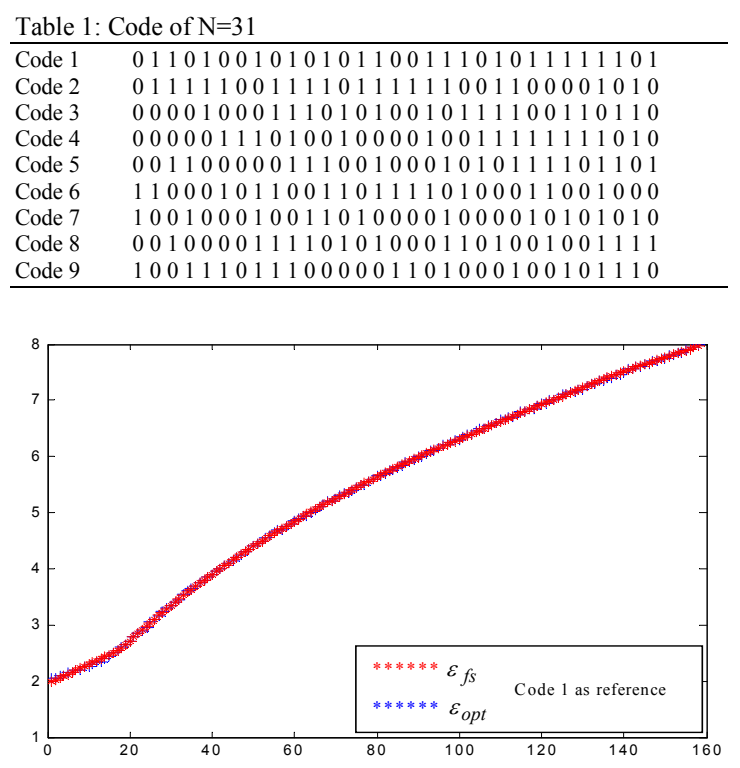

Fig. 2: The $\varepsilon_{f s}$ and $\varepsilon_{\text {opt }}$ versus their number $(\mathrm{K}=9)$

\section{RESULTS AND DISCUSSION}

In this section, we present the numerical results of our proposed method with $\mathrm{k}=9$ as number of users. The used codes in Table 1 are those of Gold having $\mathrm{N}=31$ for their good correlation properties ${ }^{[9-10]}$. Table 2 gives $\underset{\left\{v_{1}, v_{2}, v_{3}\right\}}{\stackrel{(i)}{\Gamma}}$ and $\hat{N}_{i}$ for each code and allow us to train our TS fuzzy model for different values of $k_{b}$. After learning, the TS fuzzy model generalize the relation between the optimal values of $\varepsilon\left(=\varepsilon_{\text {opt }}\right)$ and spreading code while varying $k_{b}$ values to maximize 
Table 2: Quantities $\Gamma_{\left\{v_{1}, v_{2}, v_{3}\right\}}^{(i)}$ and $\hat{N}_{i}$ of the code set having N=31

\begin{tabular}{|c|c|c|c|c|c|c|c|c|c|}
\hline code & $\begin{array}{c}\stackrel{(i)}{\Gamma} \\
\{-1,-1,-1\}\end{array}$ & 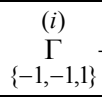 & $\begin{array}{l}\stackrel{(i)}{\Gamma} \\
+\{1,-1,-1\}\end{array}$ & $\stackrel{(i)}{\Gamma}$, & $\stackrel{(i)}{\Gamma}$ & 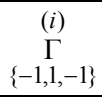 & 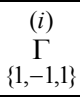 & $\begin{array}{l}(i) \\
\sum_{\{1,1,1\}}\end{array}$ & $\hat{N}_{i}$ \\
\hline 1 & 10 & 8 & & 4 & & 4 & 2 & 3 & 20 \\
\hline 2 & 2 & 4 & & 8 & & 4 & 6 & 7 & 12 \\
\hline 3 & 4 & 8 & & 8 & & 4 & 4 & 3 & 16 \\
\hline 4 & 2 & 8 & & 8 & & 2 & 2 & 9 & 12 \\
\hline 5 & 4 & 8 & & 8 & & 4 & 4 & 3 & 16 \\
\hline 6 & 2 & 8 & & 8 & & 6 & 6 & 1 & 16 \\
\hline 7 & 9 & 1( & & 6 & & 3 & 1 & 2 & 20 \\
\hline 8 & 4 & 8 & & 8 & & 4 & 4 & 3 & 16 \\
\hline 9 & 3 & 1( & & 1 & & 3 & 3 & 2 & 16 \\
\hline
\end{tabular}

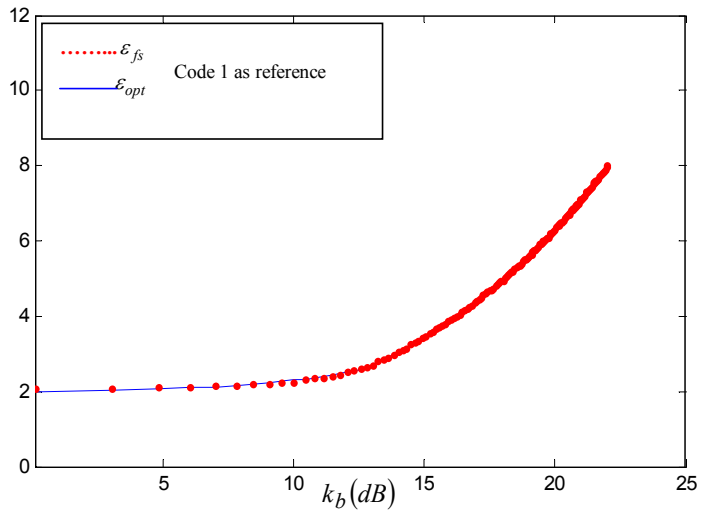

Fig. 3: The $\varepsilon_{f_{s}}$ and $\varepsilon_{\text {opt }}$ versus $k_{b}(\mathrm{~K}=9)$

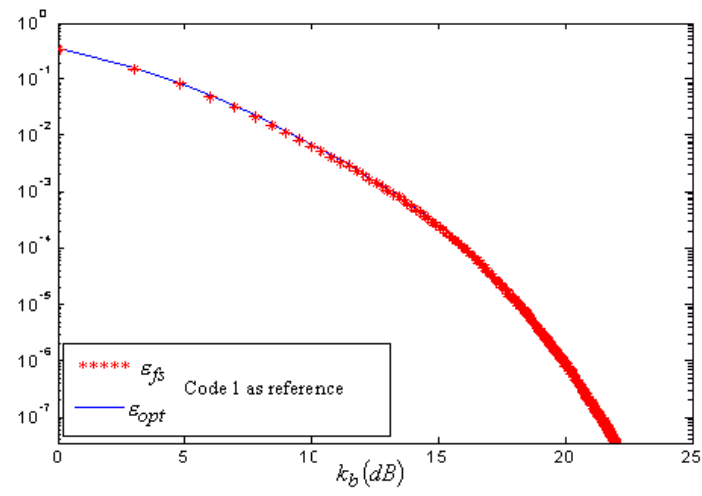

Fig. 4: BER versus $k_{b}$ for the ith user's receiver when the $\varepsilon_{f S}$ and $\varepsilon_{\text {opt }}$ are used in the performance expression, $(\mathrm{K}=9)$

SINR, it was tested with unseen $k_{b}$ values. The first code is used as reference in simulations.

In Fig. 2, we presented the evolution of the $\varepsilon_{\text {opt }}$ calculated directely by eq. (8) and eq. (9) and the $\varepsilon_{f_{s}}$ obtained using the TS fuzzy model (The number of the rules obtained is $\mathrm{c}=4$ ). We note from Fig. 2 the good agreement between both parameters $\varepsilon_{\text {opt }}$ and $\varepsilon_{f_{s}}$. Figure 3 illustrates, for different values of unseen $k_{b}$, the optimal values of the parameter $\varepsilon_{o p t}$ calculated directely by eq. (8) and eq. (9) and the results given by the TS fuzzy model $\varepsilon_{f s}$. According to this figure, we can conclude that the values obtained by the TS fuzzy model and the optimal values are identically near.

Figure 4 describes the bit error rate (BER) performances of the $i$ th user's receiver versus $k_{b}$ when the values given by the TS fuzzy model and the optimal values are used in the performance expression given by eq. (10). We remark that the BER does not undergo any degradation. It remains to note that the same results are obtained for the other codes given in Table 1. Another manner to prove the validity of our model consists to compute the RMSE ( Root Mean Square Error) of both phases: training and test. The RMSE is given by:

$R M S E=\sqrt{\frac{1}{m} \sum_{i=1}^{m}\left(\varepsilon_{\text {opt }}(i)-\varepsilon_{f_{s}}(i)\right)^{2}}$

For 500 iterations, The RMSEs were 0.015 and 0.016 for the training and test (unseen $k_{b}$ ) phases, respectively. As we do not obtain a greater error these results are in good agreement with those given on the figures.

\section{CONCLUSION}

In this study, a new method based on TakagiSugeno fuzzy systems permitted us to determine easily the optimal values of $\varepsilon$ while varying the SNR and therefore the determination of the despreading sequences weighted by stepping chip weighting waveforms for a DS/CDMA system. It is worth concluding from the numerical evaluations that we get the nearly optimal values of $\varepsilon\left(=\varepsilon_{\text {opt }}\right)$ quickly and easily by the proposed method and the bit error 
rate performance does not undergo any degradation while using the values obtained $\varepsilon_{f s}$ instead of the optimal values.

\section{REFERENCES}

1. Verdu, S., 1986. Minimum probability of error for asynchronous Gaussianmultiple-access channels. IEEE Trans. Inform.Theory., pp: 8596.

2. Jabrane, Y., R. Iqdour, B. Ait Essaid and N. Naja, 2005. Comparison of performances in cancellation of interferences in a CDMA system between two methods of detection conventional and maximum likelihood. AMSE International conference on modeling and simulation. 22- 2334 November 2005. Marrakesh, Morocco.

3. Huang, Y. and T. S. Ng, 1999. A DS-CDMA system using despreading sequences weighted by adjustable chip waveforms. IEEE Trans. Commun., 47: 1884-1896.

4. Monk, A. M., M. Davis and C. W. Helstrom, 1994. A noise-weightning approach to multiple access noise rejection -part I: theory and background. IEEE J. Select Areas. Comm., 12: 817-827.
5. Fiordaliso, A., 1999. Systèmes flous et prévision de séries temporelles. Hermes Science.

6. Bernadette, B. M. and C. Marsala, 2003. Logique floue, principes, aide à la décision. Hermes.

7. Jabrane, Y., R. Iqdour, B. Ait Essaid, N. Naja and S. Soukri, 2007. Neural Networks for Interferences suppression in DS/CDMA with Rayleigh fading channel and power control error. Science Publications JCS., 3: 174-179.

8. Jabrane, Y., R. Iqdour, B. Ait Essaid and N. Naja, 2006. Determination of weighted despreading sequences for a DS/CDMA system using a new method. IEEE section, 4th International Conference JTEA 12-14 Mai 2006, Tunisie.

9. Dinan, H. and E. B. Jabbari, 1998. Spreading codes for direct sequence CDMA and wideband CDMA cellular networks. IEEE Comm. Mag., 36: 48-54.

10. Kârkkainen, H. A. and K. P. A. Leppânen, 2000. The influence of initial-phases of a PN code set on the performance of an asynchronous DS/CDMA system. Wirless Pers.Commun., 13: 279-293. 